\title{
Article
}

\section{Performance Prediction and Design of Stratospheric Propeller}

\author{
Cong Xie, Guoquan Tao*D and Zhe Wu \\ School of Aeronautics Science and Engineering, Beihang University (BUAA), Beijing 100191, China; \\ xiecong@buaa.edu.cn (C.X.); zhewu_buaa@163.com (Z.W.) \\ * Correspondence: 09544@buaa.edu.cn
}

Featured Application: It provides a convenient propulsion force calculation method for the overall design of a stratosphere airship.

\begin{abstract}
In this paper, a performance prediction method is proposed for the design of a stratospheric propeller. The Spalart-Allmaras (S-A) model was used to calculate the airfoil performance of FX63, and the polynomial fitting method was utilized to establish the airfoil database of the lift and drag coefficient. A computational fluid dynamics (CFD) model was applied at different altitudes to prove the feasibility of the method. The CFD results were compared with the results of the vortex theory and prediction; the prediction result accuracy was improved compared with that of the vortex theory over a greater range of advance ratios. The airfoil performance data requirements and the number of iterative calculations were reduced. These results indicate that the proposed propeller design meets the requirements of stratospheric airship propulsion systems.
\end{abstract}

Keywords: performance prediction; propeller design; stratospheric propeller

check for

updates

Citation: Xie, C.; Tao, G.; Wu, Z. Performance Prediction and Design of Stratospheric Propeller. Appl. Sci. 2021, 11, 4698. https://doi.org/ 10.3390/app11104698

Academic Editor: Rosario Pecora

Received: 22 April 2021

Accepted: 19 May 2021

Published: 20 May 2021

Publisher's Note: MDPI stays neutral with regard to jurisdictional claims in published maps and institutional affiliations.

Copyright: (c) 2021 by the authors. Licensee MDPI, Basel, Switzerland. This article is an open access article distributed under the terms and conditions of the Creative Commons Attribution (CC BY) license (https:// creativecommons.org/licenses/by/ $4.0 /)$.

\section{Introduction}

The capability to fly continuously for months in the stratosphere is an elusive goal. Stratospheric airships have enormous potential for long-endurance vehicles, which do not need to stay in motion to remain in the air, since lift is generated through the buoyancy effect rather than aerodynamics. The efficient propulsion of propellers is essential for the maneuverability and sustainability of airships and cannot be achieved through turbojet and turbofan engines [1-5].

However, the environment in the stratosphere, which is above $20 \mathrm{~km}$, has a much lower air density. Additionally, the velocity of advance of a stratospheric airship is lower than $30 \mathrm{~m} / \mathrm{s}$, which is extremely low. The low Reynolds number and small advance ratio create a significant challenge for the design of an efficient propeller.

The airfoil of high-altitude vehicles was researched by Prof. Michael S. Selig [6,7]; the aerodynamic characteristics of six typical low-Reynolds-number, high-lift, and lowresistance airfoils were analyzed, and wind tunnel experiments were carried out. Angleo [8] proposed a method of designing the chord length and twist angle to maximize propeller efficiency. Xiang [9] improved Angleo's method to align it with the requirements of engineering applications.

Rankine [10] and Froude [11] first developed the theory of propellers in the 19th century, and Drzewiecki [12] proposed the blade element theory, which neglected the induced velocity of the propeller. The design method based on minimum induced losses was firstly proposed by Betz [13] and Goldstein [14]. Theodorsen's [15] research indicated that the Betz condition for minimum energy loss could also be applied in heavy disk loadings. Glauert [16] discovered an approximation of the flow around helicoidal vortex sheets, which is better if the advance ratio of the propeller is smaller, and the result improves as the number of blades increases. 
Numerical simulation technology has been widely used in propeller performance calculations with the development of computer technology. The $k-\varepsilon$ turbulence model was used by Wang [17] to simulate the performance of a high-altitude propeller, and the result was in good agreement with the scaled wind tunnel experiment. Xiang [18] proposed an improved design method of propellers, which could be optimized under specific operative condition and according to the profile distribution along the blade, and the wind tunnel test showed that the method was feasible.

Reasonable accurate performance prediction could be obtained based on vortex theory and blade element theory [19,20]. Liu [21] studied the propeller's aerodynamic characteristics utilizing the vortex theory and a propeller model [22,23], and the results indicated that the S-A model is more suitable for calculating aerodynamic parameters. The performance predicted by the vortex theory was similar to that determined by scale wind tunnel tests. However, in the design process, the airfoil data of each section are needed for calculating the performance of the propeller.

The S-A model was performed in Fluent to calculate the airfoil performance at different angles of attack and Reynolds numbers. The performance curves of the lift and drag coefficient underwent polynomial interpolation to obtain the database, which was used to calculate the performance of the propeller. The CFD model with different mesh numbers was applied to verify the mesh independence, and the CFD method at different altitudes was applied with the similarity theory to prove the feasibility of the method.

In this paper, a prediction method is proposed to reduce calculations or experimental times and to predict a propeller's performance on the basis of characteristic blade element performance. The prediction parameters are discussed, and a realistic and accurate prediction model is obtained which reduces the calculation amount with respect to the vortex theory, in regard not only to airfoil performance calculation, but also to propeller performance calculation.

\section{Methodology}

The blade element theory is typically used to predict the performance of a propeller. The blade is divided into a finite number of tiny segments called blade elements, and the aerodynamic performance of each blade element can be obtained according to the airfoil theory. The blade element is integrated along the radial direction to obtain the total aerodynamic performance of the blade.

Figure 1 presents the forces and velocities acting on the blade element, where $V_{\infty}$ is the free stream speed, $V_{a}$ is the axial induction velocity, $V_{t}$ is the tangential induction velocity, and $V$ is the total velocity of the actual airflow; $n_{s}$ is the angular velocity of the propeller, and $r$ is the radial position of the blade element; $\varphi_{0}$ is the induced angle of attack, $\varphi$ is the angle of actual airflow, $\theta$ is the pitch angle, and $\beta$ is the interference angle, which is given by:

$$
\tan \beta=\frac{V_{i}}{V_{0}}=\frac{\sqrt{V_{a}^{2}+V_{t}^{2}}}{V_{0}}
$$

The axial and tangential induction factors are defined as follows:

$$
\left\{\begin{array}{c}
a=\frac{V_{a}}{V_{\infty}} \\
a^{\prime}=\frac{V_{t}}{2 \pi n_{s} r}
\end{array}\right.
$$

The angle of actual airflow $\varphi$ is as follows:

$$
\tan \varphi=\frac{V_{\infty}+V_{a}}{2 \pi n_{s} r-V_{t}}
$$

The angle of attack $\alpha$, the velocity of the actual airflow $V$, and the local Reynolds number $R e$ are given by:

$$
\alpha=\theta-\varphi
$$




$$
\begin{gathered}
V=\sqrt{\left(V_{\infty}+V_{a}\right)^{2}+\left(2 \pi n_{s} r-V_{t}\right)^{2}}=V_{\infty} \frac{(1+a)^{2}}{\sin \varphi} \\
\operatorname{Re}=\frac{\rho V b}{\mu}
\end{gathered}
$$

The thrust and torque forces acting on the blade element are as follows:

$$
\left\{\begin{array}{l}
d T=\frac{1}{2} \rho V^{2} C_{T_{V}} b d r \\
d F=\frac{1}{2} \rho V^{2} C_{F_{V}} b d r
\end{array}\right.
$$

where $b$ is the chord length of the blade element. $C_{T_{V}}$ and $C_{F_{V}}$ are obtained from the geometric relationship shown in Figure $1 ; C_{L}$ and $C_{L}$ can be determined by $R e$ and $\alpha$.

$$
\left\{\begin{array}{l}
C_{T_{V}}=C_{L} \cos \varphi-C_{D} \sin \varphi \\
C_{F_{V}}=C_{L} \sin \varphi+C_{D} \cos \varphi
\end{array}\right.
$$

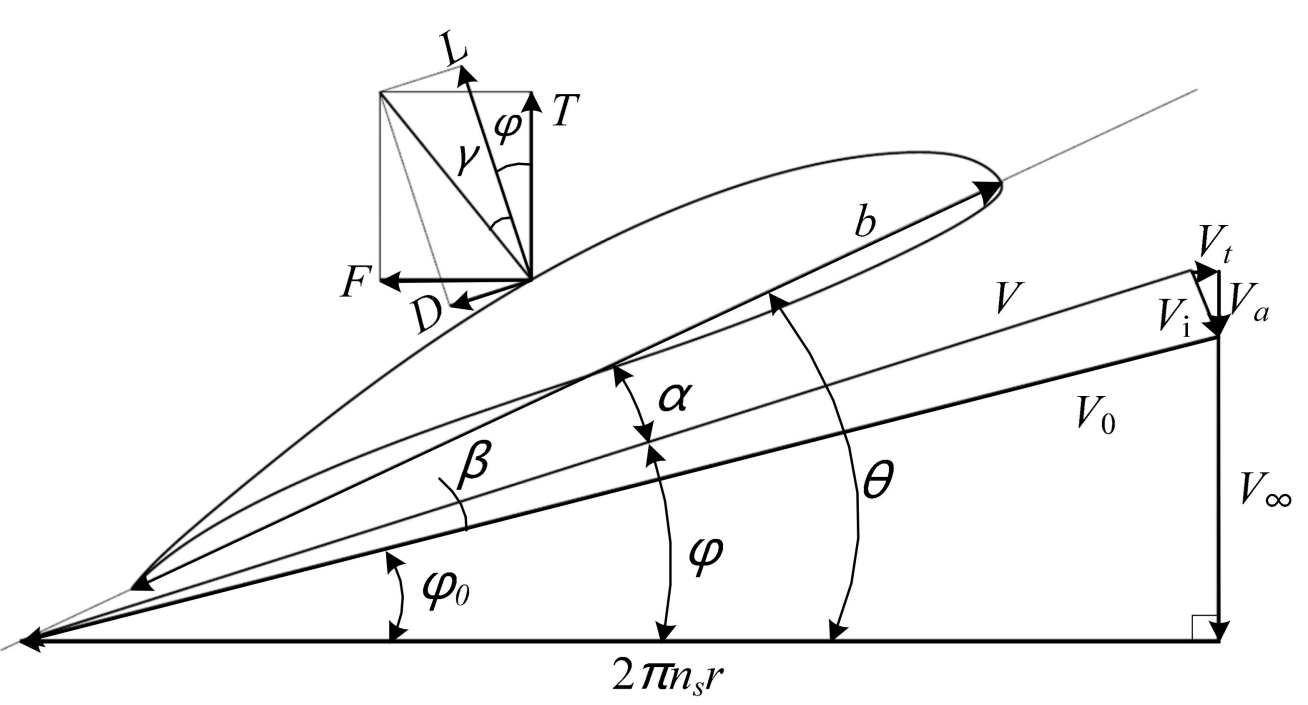

Figure 1. Forces and velocities acting on the blade element.

Equation (5) is integrated into Equation (7), and the lift and torque acting on the propeller are given by:

$$
\left\{\begin{array}{l}
d T=\frac{1}{2} \rho V_{\infty}^{2} C_{T_{V}} N_{\mathrm{b}} b \frac{(1+a)^{2}}{\sin ^{2} \varphi} d r \\
d M=r d F=\frac{1}{2} \rho V_{\infty}^{2} C_{F_{V}} N_{\mathrm{b}} b \frac{(1+a)^{2}}{\sin ^{2} \varphi} r d r
\end{array}\right.
$$

The momentum theory equations are shown below:

$$
\left\{\begin{array}{l}
d T=4 \pi r \rho V_{\infty}^{2} a(1+a) d r F \\
d M=4 \pi r^{2} \rho V_{\infty}\left(2 \pi n_{s} r\right) a^{\prime}(1+a) d r F
\end{array}\right.
$$

Combining Equations (9) and (10), we obtained:

$$
\left\{\begin{array}{c}
a=\left(\frac{4 F \sin ^{2} \varphi}{\sigma C_{T_{V}}}-1\right)^{-1} \\
a^{\prime}=\left(\frac{4 F \sin \varphi \cos \varphi}{\sigma C_{F_{V}}}+1\right)^{-1}
\end{array}\right.
$$

where $\sigma$ is the solidity ratio of the propeller, and $F$ is the Prandlt momentum loss factor.

$$
\sigma=\frac{N_{B} b}{2 \pi r}
$$




$$
F=\frac{2}{\pi} \arccos e^{-f}
$$

where $f$ is given by:

$$
f=\frac{N_{B}}{2} \frac{1-\bar{r}}{\sin \varphi_{t}}
$$

where $\varphi_{t}$ is the flow angle of the blade tip, and $\bar{r}=r / R$ is the dimensionless radius. At the beginning of the calculation, the value of $\varphi_{t}$ is unknown, and the error is not too large to replace $\varphi_{0}$.

$$
f=\frac{N_{B}}{2} \frac{1-\bar{r}}{\sin \varphi_{0}}
$$

An iterative method is used to calculate the axial and tangential induction factors $a$ and $a^{\prime}$ : (1) set the initial value of $a_{0}$ and $a_{0}{ }^{\prime}$; (2) calculate the angle of actual flow $\varphi$; (3) according to the pitch angle $\theta$, calculate the angle of attack $\alpha ;(4)$ obtain the $C_{L}$ and $C_{D}$ that form the lift and drag curves of the airfoil respectively; (5) use Equation (8) to calculate the $C_{T_{V}}$ and $C_{F_{V}}$; (6) the $a_{t}$ and $a_{t}{ }^{\prime}$ are calculated by Equation (11); (7) comparing $a_{0}$ and $a_{t}, a_{0}{ }^{\prime}$ and $a_{t}{ }^{\prime}$, if they are beyond the allowable error range, repeat steps (2) to (7); otherwise, the final $a$ and $a^{\prime}$ are generated, and the iteration is ended. The iterative process is shown in Figure 2 .

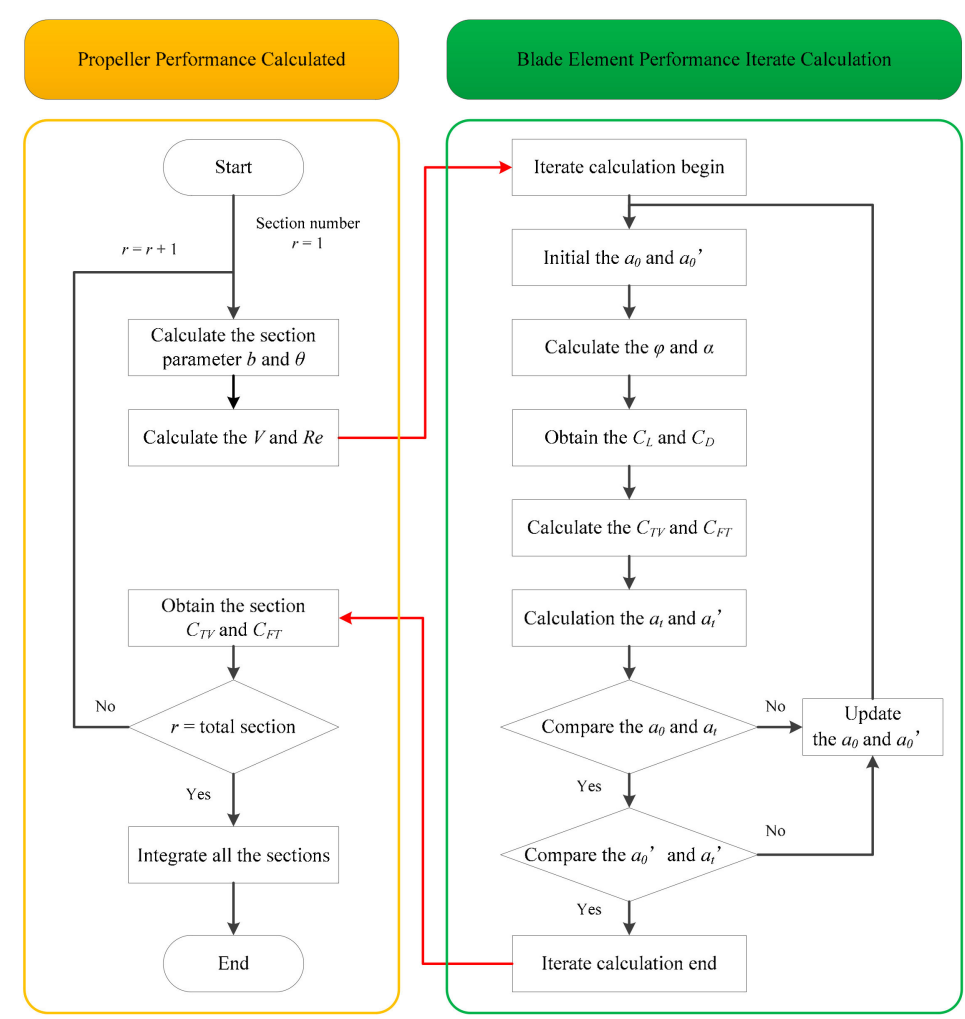

Figure 2. Iterative process for propeller performance calculation.

The thrust and torque of the propeller are obtained by:

$$
\left\{\begin{array}{l}
T=\frac{1}{2} \rho V_{\infty}^{2} N_{B} \int_{R_{h u b}}^{R} b \frac{(1+a)^{2}}{\sin ^{2} \varphi} C_{T_{V}} d r \\
M=\frac{1}{2} \rho V_{\infty}^{2} N_{B} \int_{R_{h u b}}^{R} b \frac{(1+a)^{2}}{\sin ^{2} \varphi} C_{F_{V}} r d r
\end{array}\right.
$$


The dimensionless parameter thrust coefficient $C_{T}$, the power coefficient $C_{P}$, and the efficiency $\eta$ are defined as follows:

$$
\left\{\begin{array}{c}
C_{T}=\frac{T}{\rho n_{S}^{2} D^{4}} \\
C_{P}=\frac{2 \pi s_{s} M}{\rho n_{s}^{3} D^{5}} \\
\eta=\frac{T V_{\infty}}{2 \pi n_{s} M}=\frac{C_{T}}{C_{P}} \lambda
\end{array}\right.
$$

where $\lambda$ is the advance ratio, given by:

$$
\lambda=\frac{V_{\infty}}{n_{s} D}
$$

\section{Propeller Design}

According to the propulsion system requirements of the stratospheric airship design, the design indexes of the propeller were determined and are listed in Table 1 . The required advance velocity appeared to be $20 \mathrm{~m} / \mathrm{s}$; the required thrust resulted to be larger than $100 \mathrm{~N}$, and the efficiency larger than $70 \%$.

Table 1. The design index of propeller.

\begin{tabular}{cccccc}
\hline Altitude & Velocity & Rated Speed & Rated Thrust & Diameter & Efficiency \\
\hline $20 \mathrm{~km}$ & $10 \mathrm{~m} / \mathrm{s}$ & $600 \mathrm{RPM}$ & $\geq 100 \mathrm{~N}$ & $\leq 3.5 \mathrm{~m}$ & $\geq 70 \%$ \\
\hline
\end{tabular}

A two-blade propeller was selected due to its low speed and large diameter, and the FX63 airfoil was selected for the propeller. The Mach number of the propeller tips was restricted as follows:

$$
\frac{\sqrt{\left(\pi D n_{s}\right)^{2}+V_{0}^{2}}}{c} \leq 0.7
$$

where $c$ is the local sound velocity.

The propeller geometry is defined by the pitch angle $\theta$ and the chord length $b$ along the radius of the blade from the hub to the tip, which is determined as follows:

$$
\begin{aligned}
& \theta=\theta_{\text {char }}+0.4387+0.3040 \bar{r}-3.9616 \bar{r}^{2}+5.1180 \bar{r}^{3}-1.6284 \bar{r}^{4}-0.3244 \bar{r}^{5} \\
& b=\left(0.084241-0.85789 \bar{r}+4.7176 \bar{r}^{2}-9.6225 \bar{r}^{3}+8.5004 \bar{r}^{4}-2.7959 \bar{r}^{5}\right) D
\end{aligned}
$$

where $\theta_{\text {char }}$ is the characteristic pitch angle of the propeller, $D$ is the diameter of the propeller, and $\bar{r}=0.1,0.2, \ldots, 1$.

\section{Performance Prediction}

The aerodynamic loss of the propeller is mainly concentrated on the root and tip. As the root must be strong enough to withstand loads such as the rotating centrifugal force and the bending moment of the whole propeller, it is usually too thick to exhibit good aerodynamic performance. The vortex at the tip increases the induced aerodynamic resistance and reduces the thrust, and if the rotating speed is too high, the tip is prone to generating shock loss. Therefore, the thrust is mainly generated from $0.7 R$ to $0.9 R$ of the blade. The performance of the propeller is determined among these sections, and the propeller performance can be predicted through the characteristic blade element $r_{\text {char }}$. The thrust and torque of the propeller can be given by:

$$
\left\{\begin{array}{c}
T=\left.\frac{1}{2} \rho V_{\infty}^{2} N_{B} \frac{(1+a)^{2}}{\sin ^{2} \varphi} C_{T_{V}}\right|_{r_{\text {char }}} \int_{r_{\text {char }}-r_{0}}^{r_{\text {char }}+r_{0}} d r \\
M=\left.\frac{1}{2} \rho V_{\infty}^{2} N_{B} b \frac{(1+a)^{2}}{\sin ^{2} \varphi} C_{F_{V}}\right|_{r_{\text {char }}} \int_{r_{\text {char }}-r_{0}}^{r_{\text {char }}+r_{0}} r d r
\end{array}\right.
$$


where $r_{0}$ is the prediction parameter that indicated the coverage on both sides of the characteristic blade element. In this article, $0.75 R$ is selected as the characteristic blade element. Equation (22) and the efficiency of the propeller can be given as:

$$
\left\{\begin{array}{c}
T=\left.\frac{1}{2} \rho V_{\infty}^{2} N_{B} \frac{(1+a)^{2}}{\sin ^{2} \varphi} C_{T_{V}}\right|_{0.75 R} \cdot 2 r_{0} \\
M=\left.\frac{1}{2} \rho V_{\infty}^{2} N_{B} b \frac{(1+a)^{2}}{\sin ^{2} \varphi} C_{F_{V}}\right|_{0.75 R} \cdot 1.5 R r_{0} \\
\eta=\frac{4 \lambda}{3 \pi b} \frac{\left.C_{T_{V}}\right|_{0.75 R}}{\left.C_{F_{V}}\right|_{0.75 R}}
\end{array}\right.
$$

The thrust $T$ and the torque $M$ are predicted by the $0.75 R$ element and $r_{0}$; the $0.75 R$ element performance is obtained by iterate calculation ( $r_{0}$ will be discussed later). The efficiency $\eta$ is independent of $r_{0}$ and depends on the $0.75 R$ element performance. This prediction method only needs an iterative calculation of the performance of $r_{\text {char }}$, rather than of the whole section of the propeller; a lower amount of calculation is also required to determine the airfoil performance.

\section{Finite Element Analysis}

\subsection{Airfoil Model}

ANSYS ICEM was used to generate a two-dimensional O-Block mesh of the fluid domain, in which the number of grid points was $300 \times 100$. A density-based solver was applied in ANSYS Fluent, and the Reynolds average Navier-Stokes (RANS) equation and the S-A turbulence model were adopted. The Green-Gauss node-based scheme was selected as the discretization scheme, and the second-order upwind scheme was selected for turbulence equation discretization. The computational domain was 15 times the chord length. The far-field pressure was used as the boundary condition, and the airfoil was set as the wall boundary. The residual value was required to be below $1 \times 10^{-6}$ to ensure convergence. The mesh of the airfoil is shown in Figure 3.
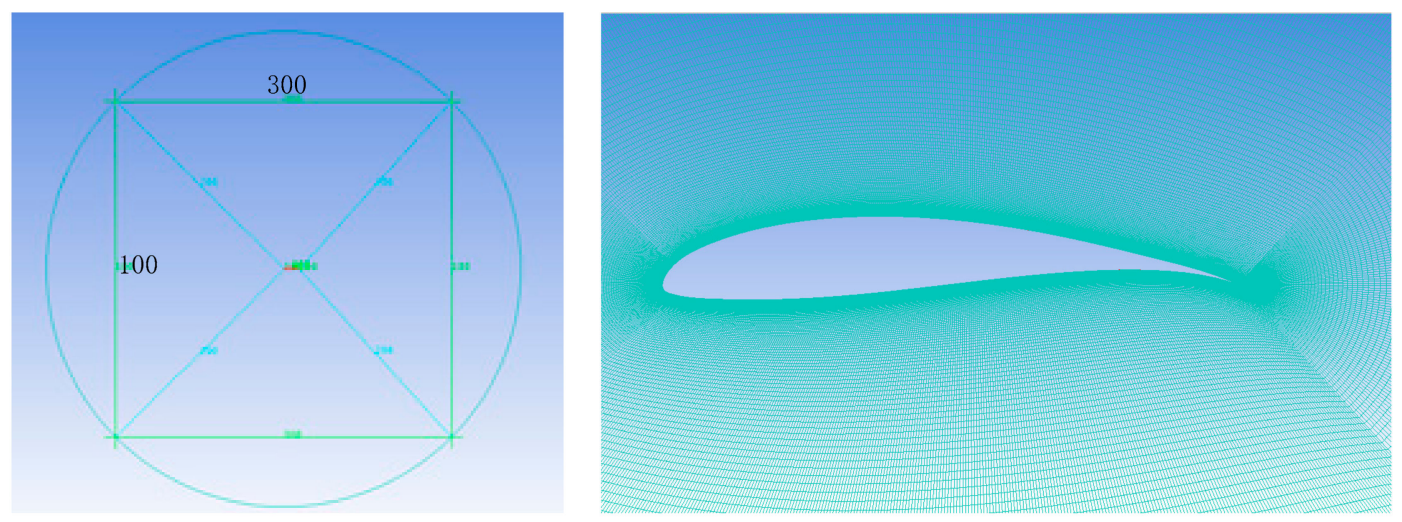

Figure 3. Mesh of the airfoil.

\subsection{Propeller Model}

A three-dimensional mesh was generated in ICEM. Figure 4 shows the computational domain, which consisted of two components, an internal rotation domain, and an external static domain. The upstream distance of the static domain was 10 times the diameter of the propeller, the downstream one was 20 times, and the diameter of the static domain was 10 times the diameter of the propeller. The rotation domain's speed is $n_{s}$, whereas the static domain is stationary. The interface boundary condition was used as the exchange data between the static and the rotation domains, the velocity inlet and pressure outlet were adopted as the boundary conditions of the static domain, and the surface of the propeller was set as the wall. 


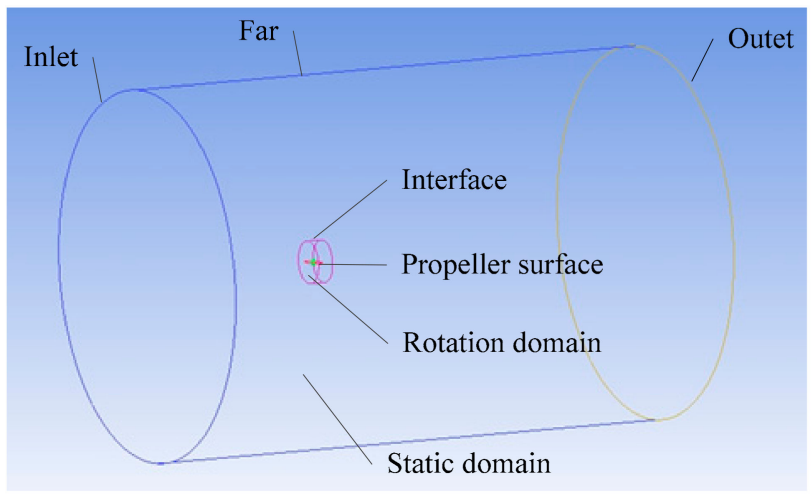

Figure 4. Domain and boundary of the propeller.

The rotational domain was composed of 5.55 million meshes, the blade surface was a triangular mesh, and the boundary layer around the surface was an hexagonal mesh, which is shown in Figure 5. A tetrahedral mesh was used to fill the space between the propeller surface and the interface.
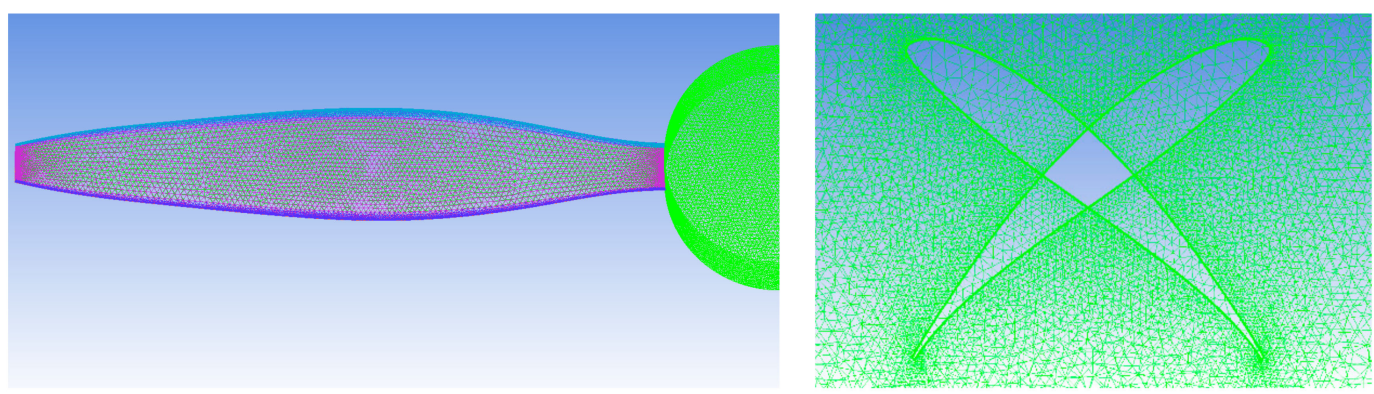

Figure 5. Mesh near the propeller.

The static domain is shown in Figure 6, which composed of 1.97 million hexagonal meshes, which were generated based on a three-dimensional O-Block.

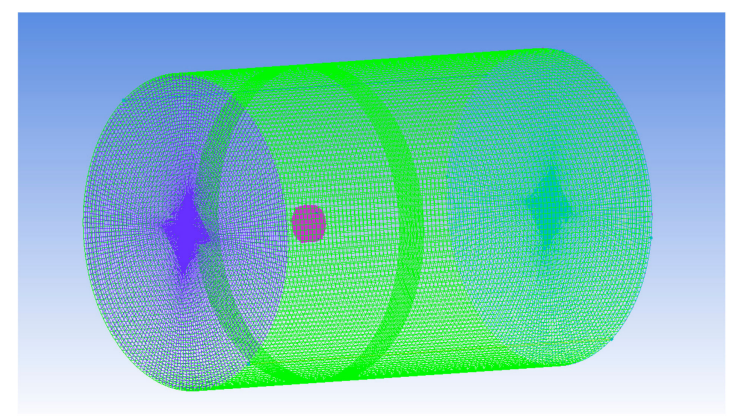

Figure 6. Mesh of the propeller.

FLUENT with a pressure-based solver was used to perform the CFD analyses. The control equation was discretized using the finite volume method, and the RANS equation and the $k-\omega$ SST Low-Re corrections turbulence model were adopted. The multiple reference frame (MRF) model was used to calculate the rotational motion of the propeller. The pressure-velocity coupling algorithm and the second-order upwind scheme were applied. The parameter $y^{+}$is the dimensionless wall distance for a wall-bounded flow, which was required to be less than 1 according to the boundary layer mesh on the propeller. The CFD results showed that $y^{+}$was approximately 0.7 along the blade length in this model. 
According to the propeller similarity theory, the performance at different altitudes should be consistent when the Reynolds number and the advance ratio are equal.

$$
\left\{\begin{array}{c}
R e=\frac{\rho_{0} n_{0} D_{0}^{2}}{\mu_{0}}=\frac{\rho_{1} n_{1} D_{1}^{2}}{\mu_{1}} \\
\lambda=\frac{V_{0}}{n_{0} D_{0}}=\frac{V_{1}}{n_{1} D_{1}}
\end{array}\right.
$$

For the same propeller model, $D_{0}=D_{1}$ the scaling ratio can be determined by:

$$
\left\{\begin{array}{c}
\eta_{\rho}=\frac{\rho_{1}}{\rho_{0}} \\
\eta_{n}=\frac{n_{1}}{n_{0}}=\frac{\rho_{0} \mu_{1}}{\rho_{1} \mu_{0}} \\
\eta_{V}=\frac{V_{1}}{V_{0}}=\frac{n_{0}}{n_{1}}
\end{array}\right.
$$

The atmospheric conditions at different altitudes are shown in Table $2 . H=20 \mathrm{~km}$ was selected as the baseline. The scaling ratio is listed in Table 3.

Table 2. Atmosphere conditions at different altitudes.

\begin{tabular}{ccccc}
\hline $\mathbf{H} / \mathbf{k m}$ & $\rho / \mathbf{k g} \cdot \mathbf{m}^{-\mathbf{3}}$ & $\mathbf{T} / \mathbf{K}$ & $\mathbf{P} / \mathbf{P a}$ & $\mu / \mathbf{k g} \cdot \mathbf{m}^{-\mathbf{1}} \cdot \mathbf{s}^{-\mathbf{1}}$ \\
\hline 15 & 0.19367 & 216.65 & $12,044.5$ & $1.4216 \times 10^{-5}$ \\
18 & 0.12164 & 216.65 & 7565.2 & $1.4216 \times 10^{-5}$ \\
20 & 0.08803 & 216.65 & 5474.9 & $1.4216 \times 10^{-5}$ \\
\hline
\end{tabular}

Table 3. Scaling ratio at different altitudes.

\begin{tabular}{ccccc}
\hline Altitude & Length & Density & Rotation Speed & Advance Speed \\
\hline 15 & 1 & 2.200 & 0.454 & 2.200 \\
18 & 1 & 1.382 & 0.724 & 1.382 \\
20 & 1 & 1 & 1 & 1 \\
\hline
\end{tabular}

\section{Result and Discussion}

\subsection{Airfoil Performance of FX-63}

The blade element performance was required to design and predict the performance of the propeller. A CFD simulation was applied to obtain the lift and drag coefficient curves of the FX63 airfoil for different Reynolds numbers and angles of attack (AOA). The range of Reynolds numbers changed from 1000 to 100,000, and the AOA changed from $-5^{\circ}$ to $15^{\circ}$. The lift and drag coefficient curves are presented in Figures 7-9.
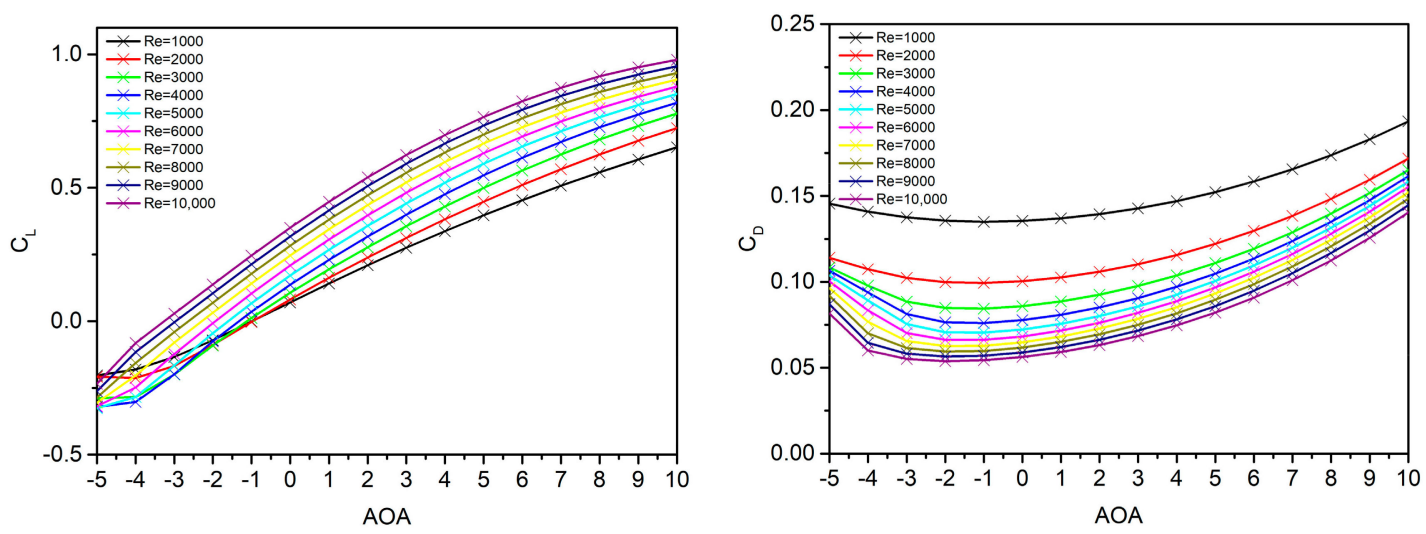

Figure 7. FX63 airfoil performance for $R e=1000-10,000$. 

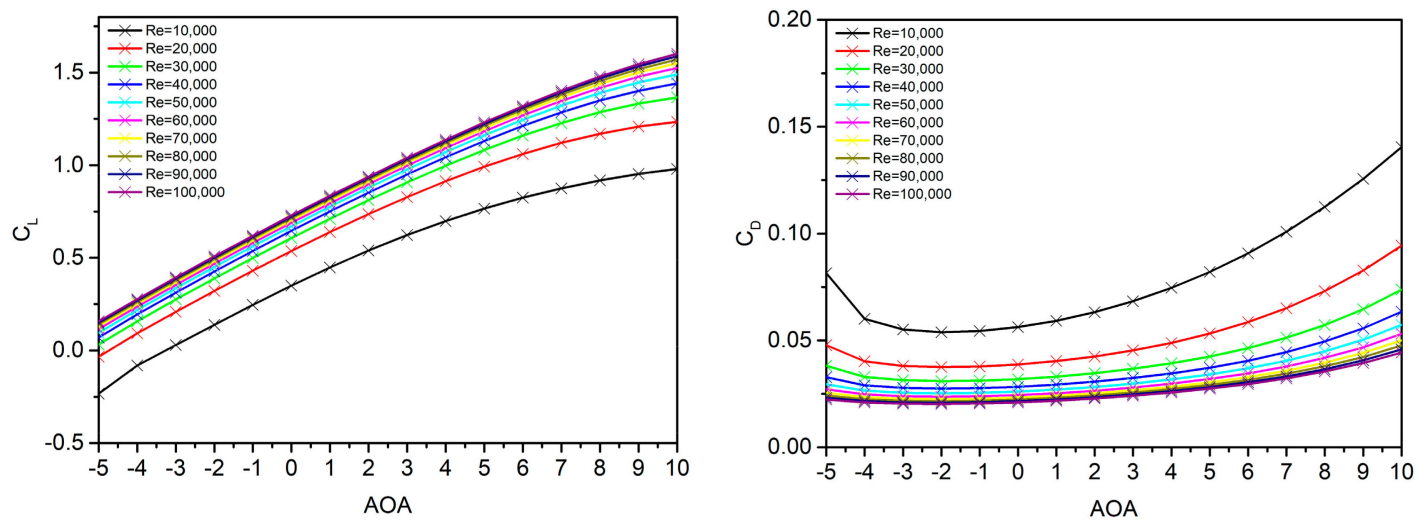

Figure 8. FX63 airfoil performance for $R e=10,000-100,000$.
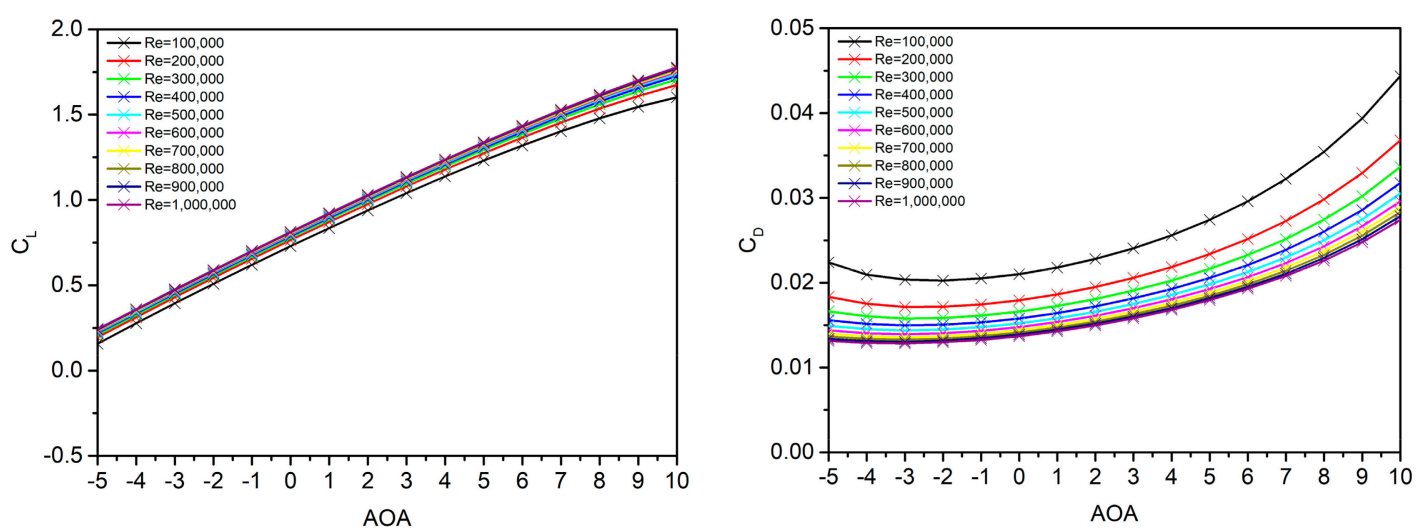

Figure 9. FX63 airfoil performance for $R e=100,000-1,000,000$.

The polynomial fitting method in MATLAB was used to interpolate the lift and drag coefficient curves, when the Reynolds number increased from 1000 to 1,000,000. The airfoil lift and drag coefficient curves at $R e=8000$ and 200,000 are presented in Figures 10 and 11, respectively. The polynomial curves were in good agreement with the CFD data. The polynomial equations are reported below the figures.

$$
\begin{aligned}
& C_{L}=\left\{\begin{array}{crl}
0.0009 \alpha^{3}+0.004 \alpha^{2}+0.114 \alpha+0.289 & -5 & \leq \alpha \leq-2 \\
-0.0003 \alpha^{3}+0.003 \alpha^{2}+0.102 \alpha+0.283 & -2 & \leq \alpha \leq 1 \\
3.8 \times 10^{-6} \alpha^{4}-4.5 \times 10^{-5} \alpha^{3}-3.7 \times 10^{-3} \alpha^{2}+0.103 \alpha+0.282 & 1 & \leq \alpha<15
\end{array}\right. \\
& C_{D}=\left\{\begin{array}{crl}
-0.001 \alpha^{3}-0.007 \alpha^{2}-0.016+0.047 & -5 \leq \alpha \leq-2 \\
-0.0001 \alpha^{3}+0.001 \alpha^{2}+0.003+0.062 & -2 \leq \alpha \leq 1 \\
4.2 \times 10^{-7} \alpha^{4}+2.1 \times 10^{-5} \alpha^{3}+3.7 \times 10^{-4} \alpha^{2}+3.3 \times 10^{-3} \alpha+0.061 & 1 \leq \alpha<15
\end{array}\right. \\
& C_{L}=\left\{\begin{array}{crl}
-0.0002 \alpha^{3}-0.003 \alpha^{2}+0.103 \alpha+0.759 & -5 & \leq \alpha \leq-2 \\
-3 \times 10^{-5} \alpha^{3}-0.001 \alpha^{2}+0.108 \alpha+0.764 & -2 \leq \alpha \leq 1 \\
4.7 \times 10^{-6} \alpha^{4}-9.3 \times 10^{-5} \alpha^{3}+6.0 \times 10^{-5} \alpha^{2}+0.104 \alpha+0.769 & 1 \leq \alpha<15
\end{array}\right. \\
& C_{D}=\left\{\begin{array}{crl}
-1.4 \times 10^{-5} \alpha^{3}+0.0001 \alpha^{2}+0.001 \alpha+0.018 & -5 \leq \alpha \leq-2 \\
-5.6 \times 10^{-6} \alpha^{3}+0.0001 \alpha^{2}+0.001 \alpha+0.018 & -2 \leq \alpha \leq 1 \\
2.0 \times 10^{-6} \alpha^{4}-3.2 \times 10^{-5} \alpha^{3}+3.0 \times 10^{-4} \alpha^{2}+0.0001 \alpha+0.018 & 1 \leq \alpha<15
\end{array}\right.
\end{aligned}
$$



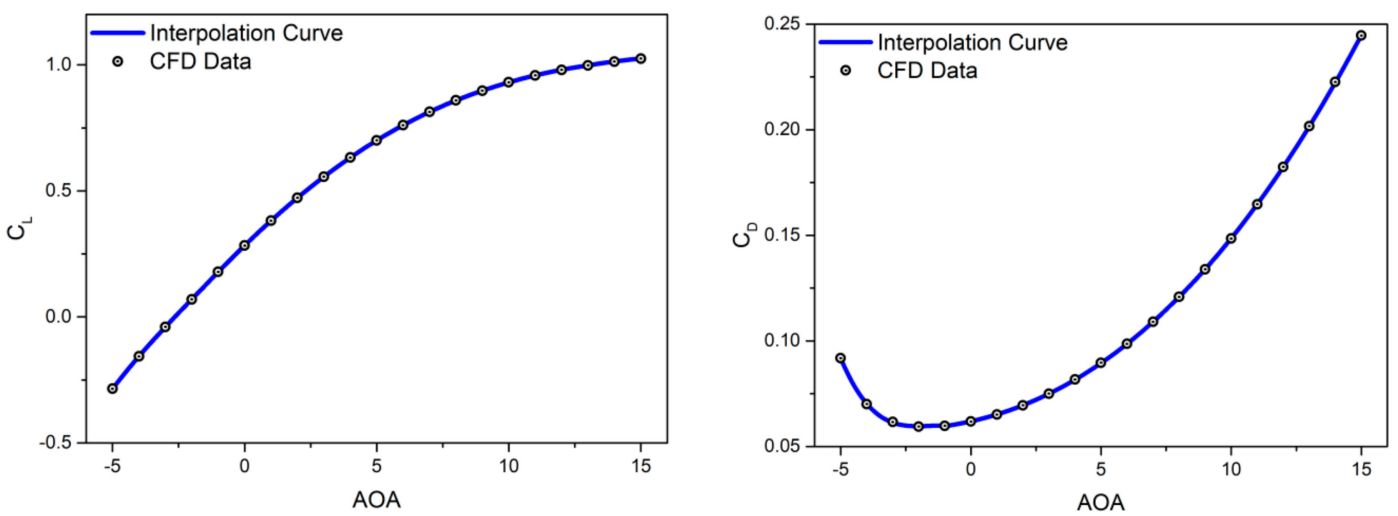

Figure 10. FX63 airfoil performance at $R e=8000$.
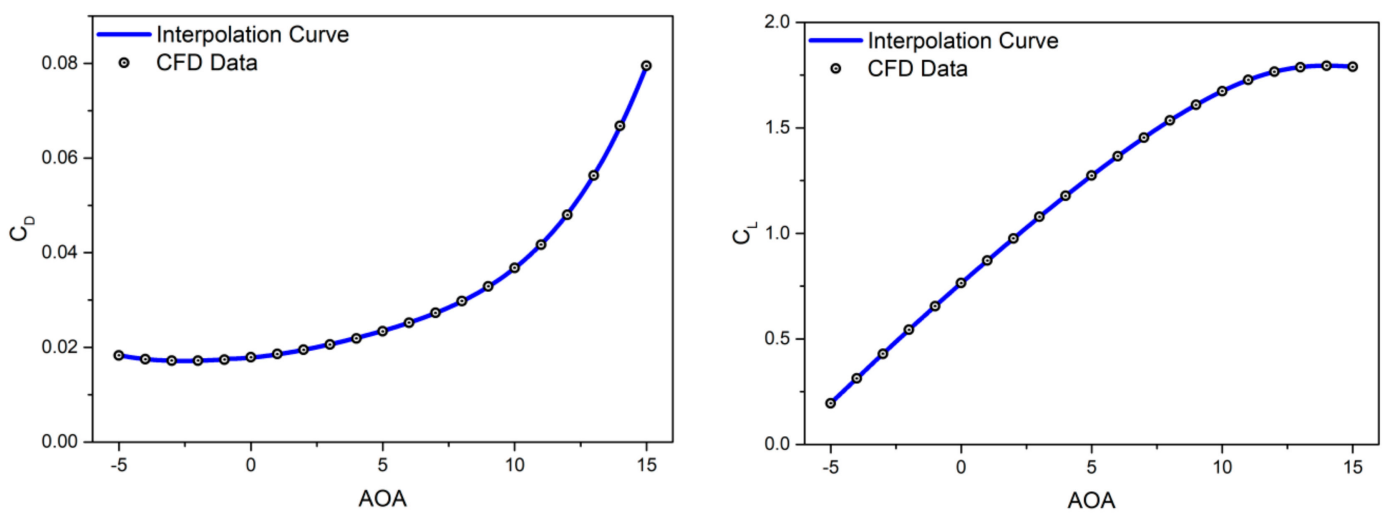

Figure 11. FX63 airfoil performance at $R e=200,000$.

\subsection{Performance of the Designed Propeller}

According to the performance calculation process, chord length $b$ and pitch angle $\theta$ are the initial input condition. In the different sections, $b$ is fixed once the diameter of the propeller is determined. To meet the rated thrust, $\theta_{\text {char }}$ is adjusted, and the iterative calculation needs to be performed with the data of airfoil performance.

The shape parameters are shown in Figure 12. The pitch angle decreased with the increase of $\bar{r}$. The chord length first increased, reached a maximum value near $0.5 R$, and then decreased. The 3D solid model of the propeller is shown in Figure 13 and was constructed according to the design parameters.

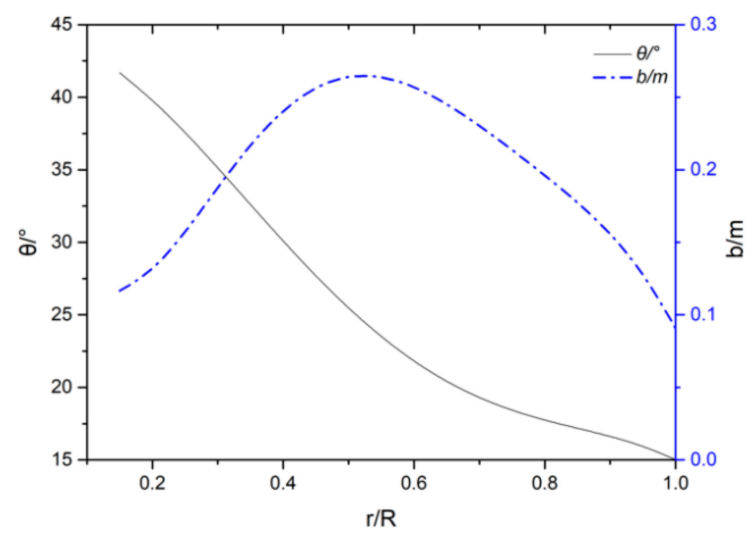

Figure 12. Shape parameters of the propeller.

Figure 14 shows the angle of attack and interference angle of the propeller, which were designed according to the vortex theory and standard strip analysis. The interference 
angle $\beta$ affects the angle of attack $\alpha$ and, consequently, the aerodynamic performance of the blade. The AOA was negative near the root, and most of the blade section showed a small AOA.
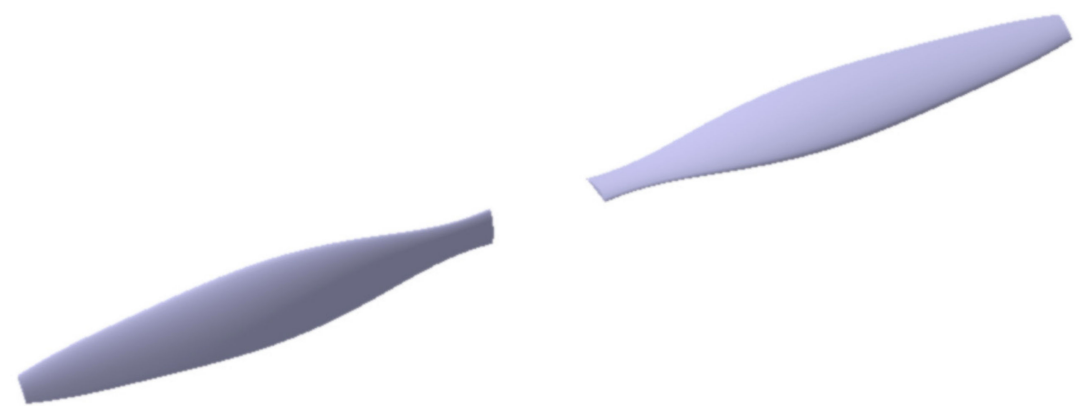

Figure 13. Solid model of the propeller.

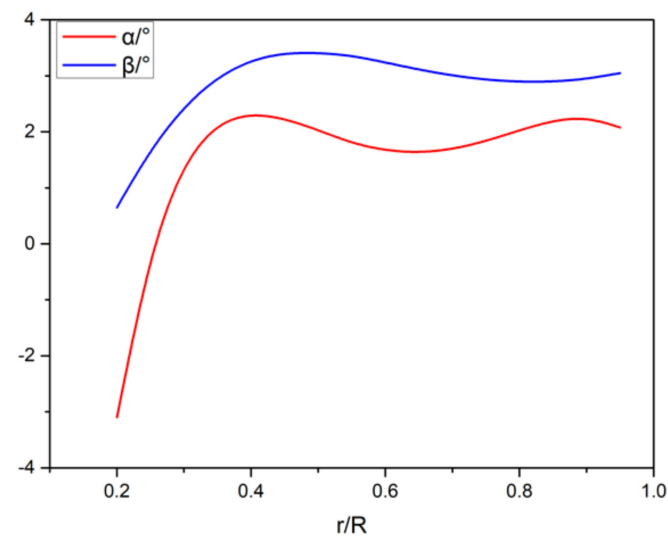

Figure 14. Angle of attack and interference angle of the propeller.

The deviation of four different mesh qualities is shown in Figure 15. The cell number of the coarse mesh was 5.65 million, the mesh used consisted of 7.52 million cells, the refined mesh comprised 10.2 million cells, and the other mesh 8.65 million cells. The maximum deviation from the mesh used was less than $1.41 \%$ for the thrust coefficient, $2.14 \%$ for the power coefficient, and $1.44 \%$ for the efficiency. The results of mesh independency verification showed that the number of cells had a little influence on the results; a mesh with 7.52 million cells was reliable and was chosen for CFD simulation in this paper.

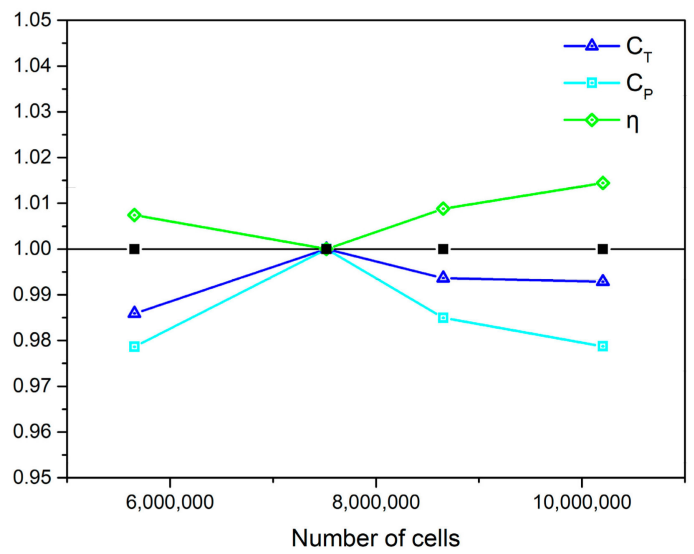

Figure 15. Mesh independency verification.

The CFD simulation at $H=15 \mathrm{~km}, 18 \mathrm{~km}$, and $20 \mathrm{~km}$ was performed using the mesh model mentioned above, and the results are shown in Figure 16. The variation tendencies 
of $C_{T}, C_{P}$, and $\eta$ were approximately the same. The $C_{T}$ and $C_{P}$ of $H=15 \mathrm{~km}$ and $H=18 \mathrm{~km}$ deviated slightly towards those of $H=20 \mathrm{~km}$ when $\lambda<0.7$, and the deviation decreased with the increase of $\lambda$. The $\eta$ values at the three altitudes were almost identical within the change range of $\lambda$. The altitude had almost no effect on the aerodynamic performance of the designed propeller, and the simulation method was reliable and correct.

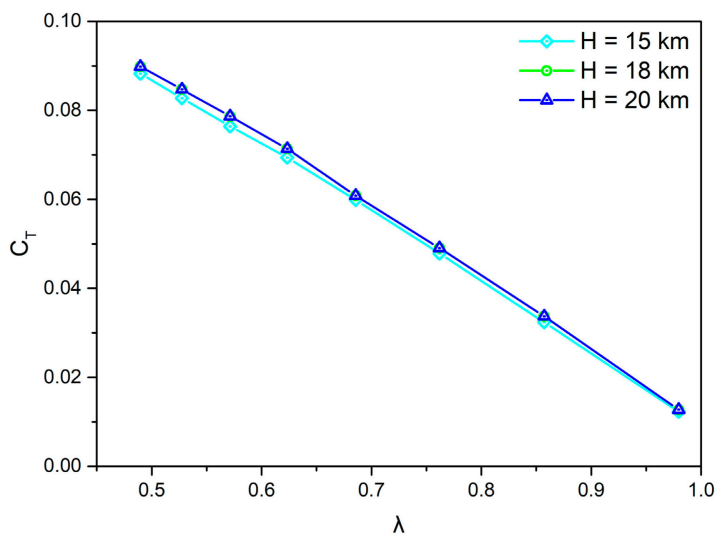

(a)

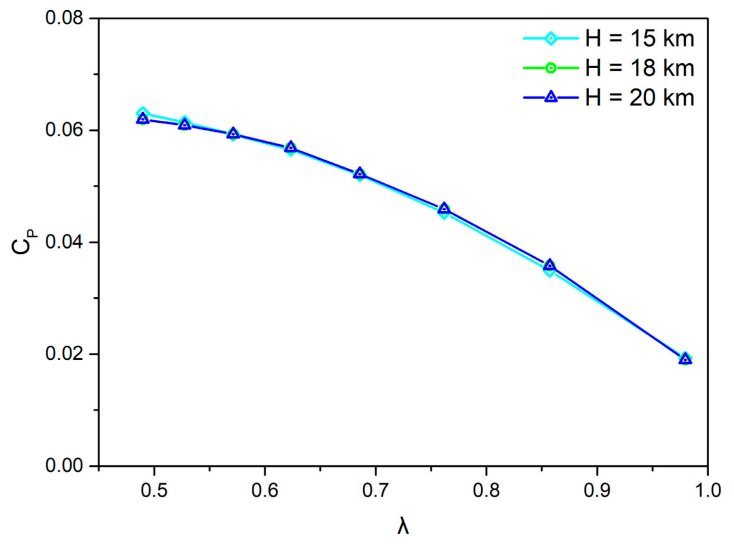

(b)

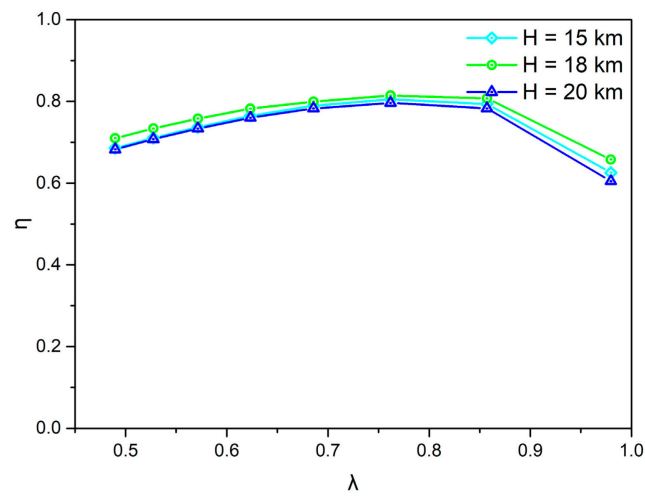

(c)

Figure 16. Propeller performance at different altitudes: (a) thrust coefficient; (b) power coefficient; (c) efficacy.

Figure 17 shows the pressure contour of the designed propeller at the design point. The pressure on the upper surface decreased from the root to the tip and from the leading edge to the trailing edge, and the minimum pressure occurred from $0.75 R$ to $0.98 R$. The pressure on the lower surface increased from root to tip and from the middle to the trailing and leading edges, and the maximum pressure occurred from $0.61 R$ to $0.99 R$.

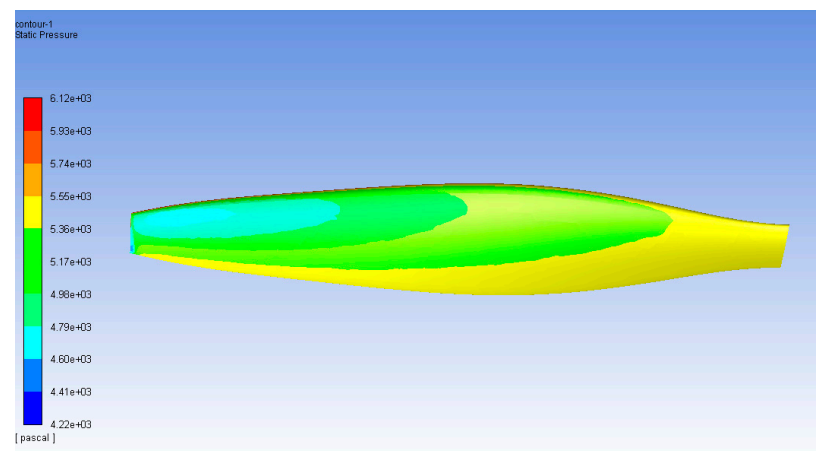

(a)

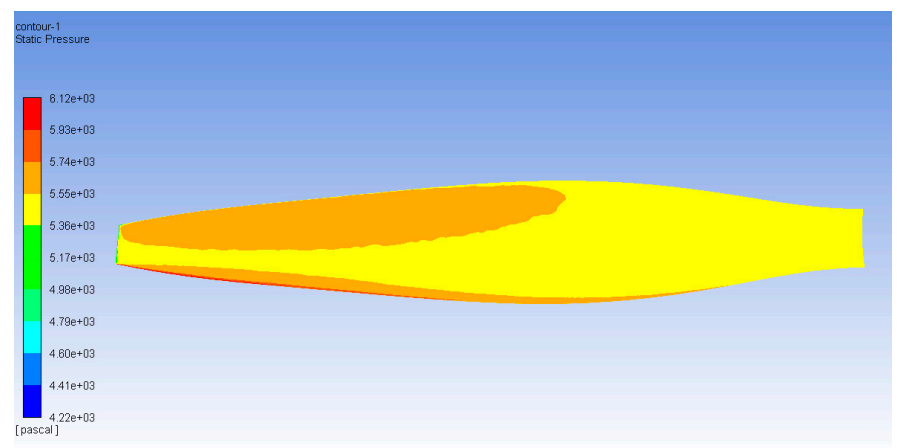

(b)

Figure 17. Pressure contour of the propeller at the design point: (a) upper surface of the blade; (b) lower surface of the blade. 


\subsection{Performance Prediction for the Designed Propeller}

The designed propeller performances calculated using the vortex theory and simulated in CFD are compared in Figure 18. The performance predicted by the vortex theory appeared in good agreement with that determined by CFD simulation. This result indicates that the prediction value of $C_{T}$ was lower than the simulation value when $\lambda<0.55$ and started to deviate from the simulation value when $\lambda>0.75$, when the deviation began to decrease. The prediction value of $C_{P}$ was higher than the simulation value when $0.65<\lambda<0.85$. The variation tendency of $\eta$ between the prediction and the simulation values was the same, and the prediction value was higher than the simulation value in the entire range of $\lambda$.

Although the vortex theory can determine the performance of the propeller, it must iteratively make computations for each section of the blade. Using the prediction method mentioned above, the aerodynamic performance of the propeller could be predicted based on the blade element performance at $0.75 R$, thus reducing the number of iterative calculations. The values $r_{0}=0.15 R, 0.2 R$, and $0.25 R$ were calculated by Equation (22) to predict the propeller performance, which meant the propeller performance was attributed to $0.6 R-0.9 R, 0.55 R-0.95 R$, and $0.5 R-R$. These results were expressed as Predict 1, Predict 2, and Predict 3, respectively.

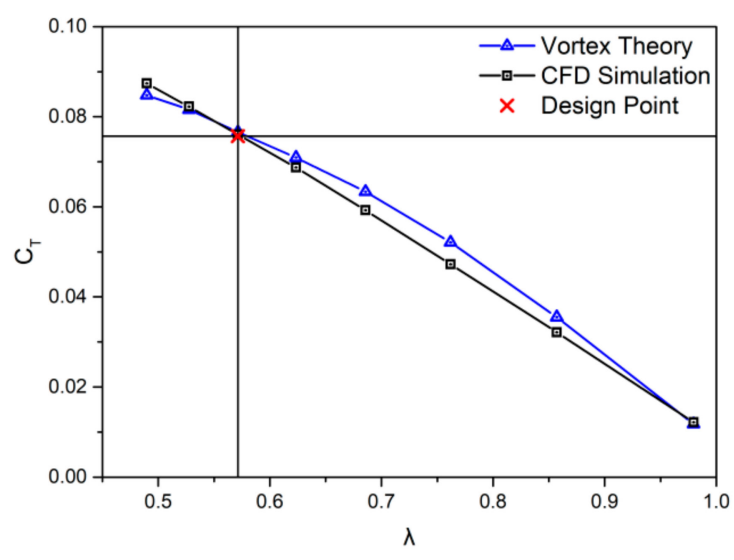

(a)

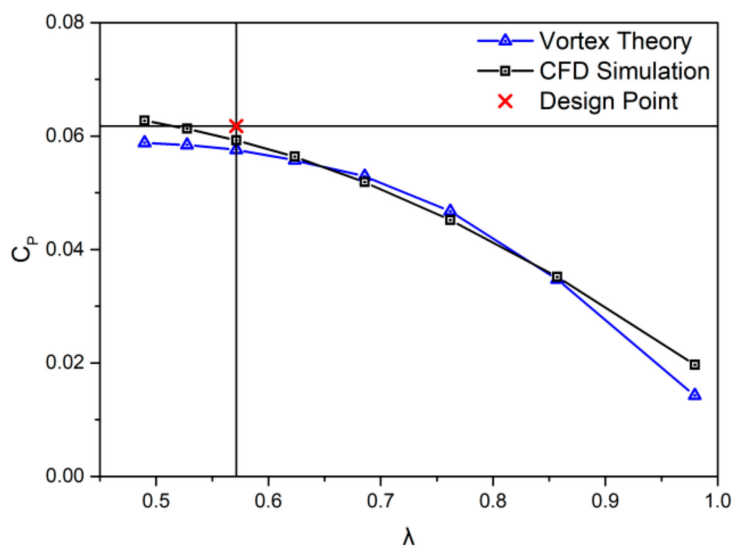

(b)

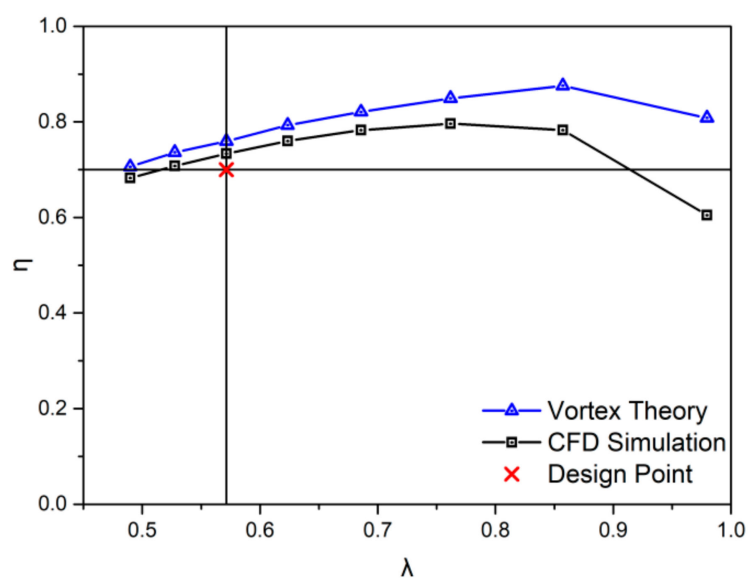

(c)

Figure 18. Comparison of the results from vortex theory with those form CFD simulation: (a) thrust coefficient; (b) power coefficient; (c) efficacy.

The propeller performances predicted based on the blade element at $0.75 R$ and simulated in CFD are compared in Figure 19. All three predictors had the same variation 
tendency as the simulation in $C_{T}, C_{P}$, and $\eta$, but the Predict 1 and Predict 2 values of $C_{T}$ and $C_{P}$ were far lower than the simulation value. The $C_{T}$ value of Predict 3 was higher than the simulation value, and the deviation decreased with increasing $\lambda$. The $C_{P}$ value of Predict 3 almost coincided with the simulation value, causing the $\eta$ value of Predict 3 to be higher than the simulation value. The $\eta$ values of the three predictions were the same according to Equation (24).

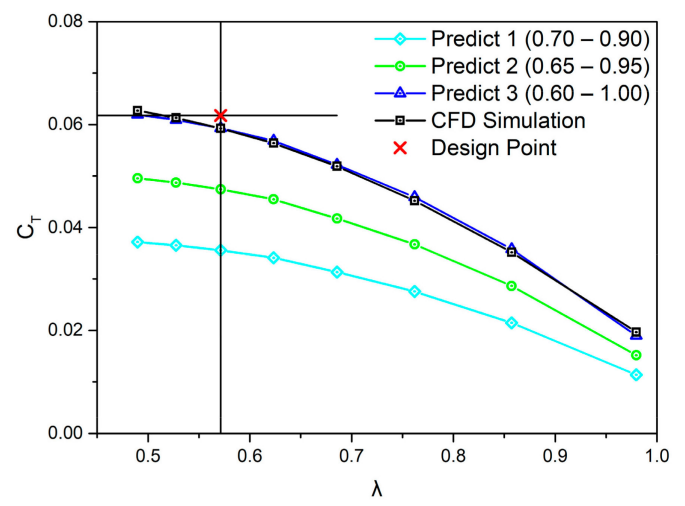

(a)

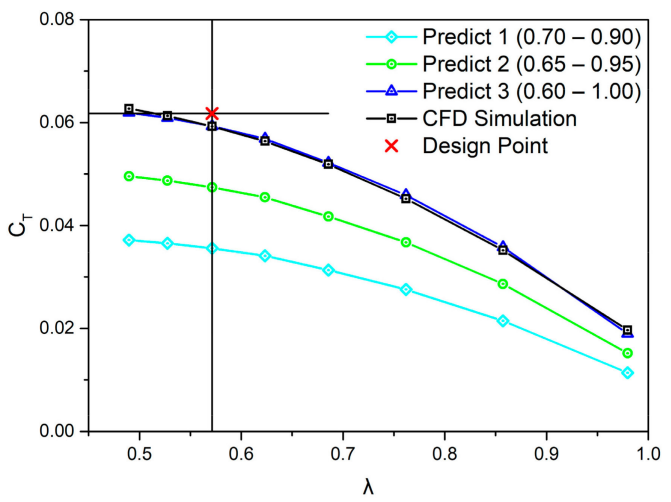

(b)

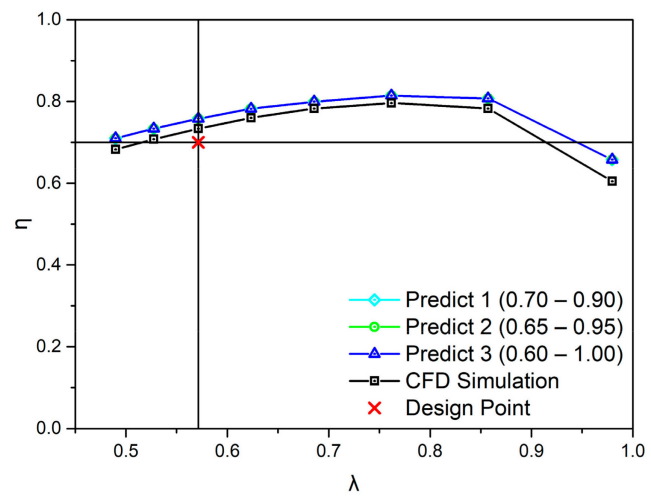

(c)

Figure 19. Comparison of predicted performance with results of CFD simulation: (a) thrust coefficient; (b) power coefficient; (c) efficacy.

Table 4 indicates that the prediction accuracy of Predict 3 was the same for the three aerodynamic parameters as that of the vortex theory at the design point. For $r_{0}=0.25 R$, Predict 3 was in accordance with the result in Figure 17. Predict 3 was more similar to the CFD simulation value within a larger $\lambda$ range, and the tendency was more consistent; $a$ higher $C_{T}$ predicted value and a lower $C_{P}$ predicted value were conducive to designing the propeller.

Table 4. Comparison of predicted value and simulate value at design point.

\begin{tabular}{cccccc}
\hline \multirow{2}{*}{$\lambda=\mathbf{0 . 5 7}$} & CFD Simulation & \multicolumn{2}{c}{ Vortex Theory } & \multicolumn{2}{c}{ Predict 3 } \\
\cline { 2 - 6 } & Value & Value & Deviation & Value & Deviation \\
\hline$C_{T}$ & 0.0760 & 0.0766 & $0.71 \%$ & 0.0787 & $3.46 \%$ \\
$C_{P}$ & 0.0592 & 0.0576 & $-2.77 \%$ & 0.0593 & $0.12 \%$ \\
$\eta$ & 0.7336 & 0.7598 & $3.57 \%$ & 0.7580 & $3.33 \%$ \\
\hline
\end{tabular}

\section{Conclusions}

In this paper, the airfoil performance of FX63 was obtained by CFD simulation, and the polynomial fitting method was utilized to establish the airfoil database of the lift and 
drag coefficients. The results indicated that the propeller design met the requirements of stratospheric airship propulsion system. The CFD model of the propeller was proven to be reliable at different altitudes with the same Reynolds number and advance ratio, and the performance curves were almost identical.

This paper proposes a method to predict the performance of a propeller for stratospheric airship based on the characteristic blade element, while reducing the amount of iterative calculations. The CFD results were compared with the results of the vortex theory and prediction; the prediction results accuracy was improved compared with that of the vortex theory over a greater range of advance ratios. The $C_{T}$ predicted deviation was $3.46 \%$, of which $C_{P}$ was $0.12 \%$, and $\eta$ was $3.33 \%$ when the character section was $0.75 R$ and the predict parameter was $0.25 R$.

This study provides a convenient propulsion force calculation method for the overall design of the stratosphere airship. The propeller performance can be predicted only by the airfoil performance of the characteristic blade element, instead of requiring calculations through an airfoil performance database. This significantly reduces the number of CFD calculations or wind tunnel tests.

Author Contributions: Conceptualization, C.X. and G.T.; Funding acquisition, G.T.; Investigation, C.X.; Methodology, G.T. and Z.W.; Project administration, Z.W.; Resources, Z.W.; Software, C.X.; Validation, G.T.; Visualization, C.X.; Writing—original draft, C.X.; Writing—review \& editing, C.X. All authors have read and agreed to the published version of the manuscript.

Funding: This research received no external funding.

Institutional Review Board Statement: Not applicable.

Informed Consent Statement: Not applicable.

Data Availability Statement: The data presented in this study are available on request from the corresponding author.

Conflicts of Interest: The authors declare no conflict of interest.

\section{References}

1. Colozza, A.; James, D. Initial Feasibility Assessment of a High Altitude Long Endurance Airship; Technical Report CR-2003-212724; Analex Corp.: Los Angeles, OH, USA, 2003.

2. D'Oliveira, F.; Melo, F.; Devezas, T. High-Altitude Platforms—Present Situation and Technology Trends. J. Aerosp. Tech. Manag. 2016, 8, 249-262. [CrossRef]

3. Zhao, D.; Liu, D. Research status, technical difficulties and development trend of stratospheric airship. Acta Aeronaut. Astronaut. Sin. 2016, 37, 45-56.

4. Wu, J.; Fang, X.D.; Wang, Z. Thermal modeling of stratospheric airships. Prog. Aerosp. Sci. 2015, 75, 26-37. [CrossRef]

5. $\mathrm{Xu}, \mathrm{Y}$; $\mathrm{Zhu}, \mathrm{W} . ; \mathrm{Li}, \mathrm{J}$. Improvement of endurance performance for high-altitude solar-powered airships: A review. Acta Astronaut. 2019, 167, 245-259. [CrossRef]

6. Selig, M.S.; Guglielmo, J.J. High-Lift Low Reynolds Number Airfoil Design. J. Aircr. 1997, 34, 72-79. [CrossRef]

7. Selig, M.S.; Mcgranahan, B.D. Wind Tunnel Aerodynamic Tests of Six Airfoils for Use on Small Wind Turbine. J. Sol. Energy 2004, 126, 986. [CrossRef]

8. D'Angelo, S.; Berardi, F.; Minisci, E. Aerodynamic Performance of Propellers with Parametric Considerations on the Optimal Design. Aeronaut. J. 2002, 106, 313-320.

9. Xiang, S.; Wang, J.; Zhang, L. A design method for high efficiency propeller. J. Aerosp. Power 2015, 30, 136-141.

10. Rankine, W.M.J. On the Mechanical Principles of the Action of the Propellers. Trans. Inst. Nav. Arch. 1865, 6, 13-30.

11. Froude, W. On the Elementary Relation between Pitch, Slip and Propulsive Efficiency; Technical Memorandum, NASA-TM-X-61726; NTRS: Washington, DC, USA, 1878; Volume 19, pp. 22-33.

12. Drzewiecki, S. Méthode pour la Détermination des Elements Mécaniques des Propulseurs Hélicoïdaux; Bulletin de l'Association Technique Maritime: Paris, France, 1892.

13. Betz, A. Schraubenpropeller mit geringstem Energieverlust. Mit einem Zusatz von 1. Prandtl. Gött. Nachr. 1919, 3, $193-217$.

14. Goldstein, S. On the vortex theory of screw propellers. Proc. R. Soc. A Math. Phys. Eng. Sci. 1929, 123, 440-465.

15. Theodorsen, T. Theory of Propellers; McGraw-Hill Book Company: New York, NY, USA, 1948.

16. Glauert, H. Airplane Propellers, Aerodynamic Theory; Division L; Durand, W.F., Ed.; Springer: New York, NY, USA, 1963; Volume IV, pp. 169-360. 
17. Wang, Y.; Liu, Z.; Tao, G. Numerical simulation of a high altitude propeller's aerodynamic characteristics and wind tunnel test. J. Beihang Aeronaut. Astronaut. 2013, 39, 1102-1105.

18. Xiang, S.; Liu, Y.-q.; Tong, G. An improved propeller design method for the electric aircraft. Aerosp. Sci. Tech. 2018, 78, 488-493. [CrossRef]

19. Nigam, N.; Tyagi, A.; Chen, P. Multi-fidelity multi-disciplinary propeller/rotor analysis and design. In Proceedings of the 53rd AIAA Aerospace Sciences Meeting, Kissimmee, FL, USA, 5 January 2015.

20. Morgado, J. Validation of New Formulations for Propeller Analysis. J. Propul. Power 2015, 31, 467-477. [CrossRef]

21. Liu, X.; He, W. Performance Calculation and Design of Stratospheric Propeller. IEEE Access 2017, 5, 14358-14368. [CrossRef]

22. Liu, P.; Ma, R.; Duan, Z. Ground Wind Tunnel Test Study of the Propeller of Stratospheric Airships. J. Aerosp. Power 2011, 26, 1775-1781.

23. Liu, P.; Duan, Z.; Ma, L. Aerodynamics properties and design method of high efficiency-light propeller of stratospheric airships. In Proceedings of the 2011 International Conference on Remote Sensing, Environment and Transportation Engineering, Nanjing, China, 24-26 June 2011. 